\title{
Simultaneous Input and State Estimation for Linear Continuous-Time Systems: A System Augmentation Approach
}

\author{
Chien-shu Hsieh \\ Department of Electrical and Electronic Engineering, Ta Hwa University of Science and Technology, Taiwan, ROC
}

\begin{abstract}
This paper presents an augmented state observer design for linear time-varying continuous-time systems with unknown inputs. An equivalent augmented state system, which is free of unknown inputs, is derived for the purpose of simultaneous input and state estimation (SISE). It is shown that, through the proposed system augmentation approach, the observer design for SISE problems can be easily solved using well-known techniques. The existence condition of SISE designs is also proposed. An illustrative example is given to show the effectiveness of the proposed results.
\end{abstract}

Keywords-unbiased minimum-variance estimation; unknown input filtering; optimal input and state estimation; system augmentation

\section{INTRODUCTION}

Simultaneous input and state estimation (SISE) has received much research attention due to its vast applications in different research areas, e.g., input reconstruction [1], [2], input estimation in physiological systems [3], fault diagnosis [4], [5], robust state estimation [6]-[8], and descriptor state estimation [9]. Most of the above work are focused on either discrete-time systems or time-invariant continuous-time systems, and very few addressed for time-varying continuoustime systems. In this paper, we devote to solve the SISE problem for linear time-varying continuous-time systems, where unknown inputs enter into both the system and the measurements.

A related work to solve the SISE problem for continuoustime systems, denoted as the CSISE problem, is to design unknown input observers (UIOs), which are free of unknown inputs. In this regards, both the reduced-order observer [10][12] and the full-order observer [1], [11]-[14] were proposed. It should be noticed that, most of the above work are focused on robust state estimation; only the work of [1] and [14] explicitly estimate the unknown inputs. Furthermore, a common approach to facilitate the above UIOs design is to use state transformation techniques or complex matrix equations solving. On the other hand, in the present paper we adopt a straightforward but more compact method, which is originated from the discrete-time unknown input filtering (UIF) [7]-[9], to propose a system augmentation approach to solve the SISE problem. Unlike those mentioned above, where the estimators for the state and unknown inputs are derived independently, the proposed new method determines the augmented state estimator which jointly estimates the state and unknown inputs estimates. This may have some benefits for the SISE problems dedicated for time-varying continuous-time systems with unknown inputs because no transformation matrices are used.

We consider linear time-varying systems of the form:

$$
\begin{gathered}
\dot{x}=A x+B u+G d \\
\mathrm{y}=C x+H d
\end{gathered}
$$

where $x \in R^{n}$ is the state vector, $u \in R^{l}$ is the control input, $d \in R^{m}$ is an unknown input vector, and $\mathrm{y} \in R^{p}$ is the measurement vector. $A, B, G, C$, and $H$ are known timevarying matrices of appropriate dimensions. Without loss of generality, we assume that matrix $C$ has full-row rank. Notice that, it may not be any knowledge concerning the model of the unknown input vector.

The main aim of the paper is to propose a simultaneous input and state observer (SISO) design which can achieve the CSISE, i.e., the SISO can yield both the system state and the unknown inputs estimates.

\section{Equivalent Augmented StATE System}

The main idea to solve the CSISE problem is to apply the descriptor system reformation [9] to the original system in order to derive an equivalent augmented state system [12] which is free of unknown inputs.

First, decompose the unknown input vector as follows [7]:

$$
d=H^{+} H d+\Pi
$$

where $M^{+}$is the Moore-Penrose pseudo-inverse of $M$ and $\Pi=$ $I-H^{+} H$. Denote the full-rank factorizations of $H$ and $\Pi$ as $H=\bar{H} \widetilde{H}$ and $\Pi=\bar{\Pi} \widetilde{\Pi}$, where $\bar{H}$ and $\bar{\Pi}$ are of full-column rank and $\widetilde{H}$ and $\widetilde{\Pi}$ are of full-row rank. Thus, (3) can be rewritten as follows:

$$
d=\widetilde{H}^{+} \bar{d}+\bar{\Pi} \tilde{d}
$$

where $\bar{d}=\widetilde{H} d$ and $\tilde{d}=\widetilde{\Pi} d$. Using (4) in the system (1), (2) yields

$$
\begin{gathered}
\dot{x}=A x+B u+G \widetilde{H}^{+} \bar{d}+G \bar{\Pi} \tilde{d} \\
\mathrm{y}=C x+\bar{H} \bar{d}
\end{gathered}
$$


Second, defining an augmented state $\mathrm{X}=\left[\begin{array}{lll}x^{T} & \bar{d}^{T} & \tilde{d}^{T}\end{array}\right]^{T}$, system (5), (6) can be expressed as the following descriptor system:

$$
\begin{gathered}
{\left[\begin{array}{ccc}
I & 0 & 0
\end{array}\right] \dot{X}=\left[\begin{array}{ccc}
A & \mathrm{G} \widetilde{H}^{+} & \mathrm{G} \bar{\Pi}
\end{array}\right] X+B u=\tilde{A} X+B u} \\
\mathrm{y}=\left[\begin{array}{lll}
C & \bar{H} & 0
\end{array}\right] X=\breve{C} X
\end{gathered}
$$

Note that, the descriptor system dynamics (7) can be further rewritten as the following standard system dynamics:

$$
\dot{X}=\left[\begin{array}{c}
\tilde{A} \\
0 \\
0
\end{array}\right] X+\left[\begin{array}{l}
\mathrm{B} \\
0 \\
0
\end{array}\right] u+\left[\begin{array}{cc}
0 & 0 \\
I & 0 \\
0 & I
\end{array}\right]\left[\begin{array}{c}
\dot{\bar{d}} \\
\dot{\tilde{d}}
\end{array}\right]
$$

Third, using (8) and (9) the derivatives of measurements are given as follows:

$$
\dot{\mathrm{y}}=\tilde{C} X+C B u+\bar{H} \dot{\bar{d}}
$$

where

$$
\tilde{C}=\left[\begin{array}{ll}
\dot{C}+C A & \dot{H}+C G \widetilde{H}^{+} \quad \mathrm{CG} \bar{\Pi}
\end{array}\right]
$$

Solving (10) for $\dot{\bar{d}}$ by using the well-known matrix equation solution theory, one obtains

$$
\dot{\bar{d}}=\bar{H}^{+}(\dot{\mathrm{y}}-\tilde{C} X-C B u)
$$

Using (12) in (9) yields

$$
\begin{gathered}
\dot{X}=\left[\begin{array}{c}
\tilde{A} \\
-\bar{H}^{+} \tilde{C} \\
0
\end{array}\right] X+\left[\begin{array}{c}
\mathrm{B} \\
-\bar{H}^{+} C B \\
0
\end{array}\right] u+\left[\begin{array}{c}
0 \\
\bar{H}^{+} \\
0
\end{array}\right] \dot{\mathrm{y}}+\left[\begin{array}{l}
0 \\
0 \\
I
\end{array}\right] \dot{\tilde{d}} \\
=\bar{A} X+\bar{B} u+E \dot{y}+F \dot{\tilde{d}}
\end{gathered}
$$

Note that, if matrix $H$ is of full-column rank one has $\bar{\Pi}=0$, which signifies that the vector $\tilde{d}$ is null. Thus, the dedicate equivalent augmented state system is readily obtained by using the following settings in (29), where

$$
\breve{A}=\bar{A}, \breve{B}=\bar{B}, \breve{u}=u, \breve{E}_{1}=0, \breve{E}_{2}=E, \dot{\grave{y}}=\dot{y}
$$

Next, we intend to determine the unknown input vector $\dot{\tilde{d}}$ in (13) for $\bar{\Pi} \neq 0$. Using (12) in (10) yields

$$
\overline{\mathrm{y}} \equiv\left(I-\bar{H} \bar{H}^{+}\right) \dot{y}=\bar{C} X+\bar{D} u
$$

where

$$
\bar{C}=\left(I-\bar{H} \bar{H}^{+}\right) \tilde{C}, \bar{D}=\left(I-\bar{H} \bar{H}^{+}\right) C B
$$

Using (13) and (15), we obtain the following derivatives:

$$
\begin{aligned}
\overline{\mathrm{y}}^{(1)} \equiv \dot{\bar{y}}=\left(I-\bar{H} \bar{H}^{+}\right) \ddot{y}+\frac{d}{d t}\left(I-\bar{H} \bar{H}^{+}\right) \dot{y} \\
=\bar{C}_{1} X+\bar{D}_{1} \breve{u}_{1}+E_{1} \breve{y}_{1}+S_{1} \dot{\tilde{d}}
\end{aligned}
$$

where

$$
\begin{gathered}
\bar{C}_{1}=\dot{\bar{C}}+\bar{C} \bar{A}, \bar{D}_{1}=\left[\begin{array}{ll}
\dot{\bar{D}}+\bar{C} \bar{B} & \bar{D}
\end{array}\right] \\
\breve{u}_{1}=\left[\begin{array}{cc}
u^{T} & \dot{u}^{T}
\end{array}\right]^{T}, E_{1}=\bar{C} E, \breve{y}_{1}=\dot{y} \\
\mathrm{~S}_{1}=\overline{\mathrm{C}} \mathrm{F}=\left(\mathrm{I}-\overline{\mathrm{H}} \overline{\mathrm{H}}^{+}\right) \mathrm{CG} \bar{\angle} 1
\end{gathered}
$$

If $S_{1}=0$, one needs a further differentiation of $\dot{\bar{y}}$ in order to possibly determine $\dot{\tilde{d}}$. The above process will continue until there exists a full rank $S_{i}(i \geq 2)$ which satisfies the following relationship:

$$
\overline{\mathrm{y}}^{(\mathrm{i})}=\overline{\mathrm{C}}_{\mathrm{i}} \mathrm{X}+\overline{\mathrm{D}}_{\mathrm{i}} \breve{\mathrm{u}}_{\mathrm{i}}+\mathrm{E}_{\mathrm{i}} \breve{\mathrm{y}}_{\mathrm{i}}+\mathrm{S}_{\mathrm{i}} \dot{\tilde{\mathrm{d}}}
$$

where $\bar{y}^{(i)}$ is the differentiation of $\bar{y}^{(i-1)}$,

$$
\begin{gathered}
\overline{\mathrm{C}}_{\mathrm{i}}=\dot{\overline{\mathrm{C}}}_{\mathrm{i}-1}+\overline{\mathrm{C}}_{\mathrm{i}-1} \overline{\mathrm{A}}, \quad \mathrm{S}_{\mathrm{i}}=\overline{\mathrm{C}}_{\mathrm{i}-1} \mathrm{~F}, \\
\overline{\mathrm{D}}_{\mathrm{i}}=\left[\begin{array}{lll}
\overline{\mathrm{C}}_{\mathrm{i}-1} \overline{\mathrm{B}} & \dot{\overline{\mathrm{D}}}_{\mathrm{i}-1} & \overline{\mathrm{D}}_{\mathrm{i}-1}
\end{array}\right] \\
\mathrm{E}_{\mathrm{i}}=\left[\begin{array}{lll}
\overline{\mathrm{C}}_{\mathrm{i}-1} \mathrm{E} & \dot{\mathrm{E}}_{\mathrm{i}-1} & \mathrm{E}_{\mathrm{i}-1}
\end{array}\right] \\
\breve{\mathrm{u}}_{\mathrm{i}}=\left[\begin{array}{lll}
\mathrm{u}^{\mathrm{T}} & \breve{\mathrm{u}}_{\mathrm{i}-1}^{\mathrm{T}} & \dot{\mathrm{u}}_{\mathrm{i}-1}^{\mathrm{T}}
\end{array}\right]^{\mathrm{T}} \\
\breve{\mathrm{y}}_{\mathrm{i}}=\left[\begin{array}{lll}
\dot{\mathrm{y}}^{\mathrm{T}} & \breve{\mathrm{y}}_{\mathrm{i}-1}^{\mathrm{T}} & \dot{\mathrm{y}}_{\mathrm{i}-1}^{\mathrm{T}}
\end{array}\right]^{\mathrm{T}}
\end{gathered}
$$

Using (18), (22), and the fact that $S_{i}$ is of full-column rank, where $i \geq 1$, the solution of $\dot{\tilde{d}}$ is obtained as follows:

$$
\dot{\tilde{d}}=\left(S_{i}\right)^{+}\left(\overline{\mathrm{y}}^{(i)}-\bar{C}_{i} X-\bar{D}_{i} \breve{u}_{i}-E_{i} \breve{y}_{i}\right)
$$

Note that, if there is no dedicate solution of $S_{i}$ that satisfies (22) the CSISE problem cannot be solved (an illustration to remedy this problem will be given in Section IV).

Finally, using (28) in (13) yields the equivalent augmented state system of the original system (1), (2) which is free of unknown inputs as follows:

$$
\begin{gathered}
\dot{X}=\breve{A X}+\breve{B} \breve{u}+\breve{E}_{1} \breve{y}_{i}+\breve{E}_{2} \dot{\breve{y}} \\
y=\breve{C X}
\end{gathered}
$$

where

$$
\begin{aligned}
& \breve{\mathrm{A}}=\overline{\mathrm{A}}-\mathrm{F}\left(\mathrm{S}_{\mathrm{i}}\right)^{+}\left(\dot{\overline{\mathrm{C}}}_{\mathrm{i}}+\overline{\mathrm{C}}_{\mathrm{i}} \overline{\mathrm{A}}\right)
\end{aligned}
$$

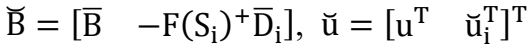

$$
\begin{aligned}
& \breve{\mathrm{E}}_{1}=-\mathrm{F}\left(\mathrm{S}_{\mathrm{i}}\right)^{+} \mathrm{E}_{\mathrm{i}}, \breve{\mathrm{E}}_{2}=\left[\begin{array}{ll}
\mathrm{E} & \mathrm{F}\left(\mathrm{S}_{\mathrm{i}}\right)^{+}
\end{array}\right]
\end{aligned}
$$




$$
\breve{y}=\left[\begin{array}{ll}
y^{T} & \left(\bar{y}^{(i-1)}\right)^{T}
\end{array}\right]^{T}
$$

Remark 1: Expending (29), we can obtain the following fictitious unknown inputs dynamics:

$$
\left[\begin{array}{c}
\dot{\bar{d}} \\
\dot{\tilde{d}}
\end{array}\right]=\left[\begin{array}{ll}
0 & I
\end{array}\right]\left(\breve{A} X+\breve{B} \breve{u}+\breve{E}_{1} \breve{y}_{i}+\breve{E}_{2} \dot{\breve{y}}\right)
$$

\section{Simultaneous InPUT AND State OBSERVER Design}

Based on the proposed equivalent augmented state system in Section II, the CSISE solving problem remains to find an optimal estimator for $X$. This is addressed as below.

\section{A. Existence Conditions}

This subsection examines the existence conditions of simultaneous input and state observers. From (4)-(5) and (22), we obtain that the system given by (29), (30) is equivalent to the system (1), (2) in the sense of observer design provided that matrix $S_{i}$ is of full-column rank or $\bar{\Pi}=0$. Thus, we have the following theorem.

Theorem 1: There exists a SISO if and only if

$$
\begin{aligned}
& \rho \alpha v \kappa\left[G\left(I-H^{+} H\right)\right]=m-\rho \alpha v \kappa[H] \\
& \rho \alpha v \kappa\left[\begin{array}{cc}
H & C G \\
0 & H
\end{array}\right]=\rho \alpha v \kappa[H]+\rho \alpha v \kappa\left[\begin{array}{l}
G \\
H
\end{array}\right]
\end{aligned}
$$

and the pair $\{\breve{A}, \breve{C}\}$ is detectable.

Proof: Using the identity $I-\bar{H} \bar{H}^{+}=I-H H^{+}$and the fact that matrix $\widetilde{\Pi}$ is of full-row rank, we have the following relationships:

$$
\begin{gathered}
\operatorname{rank}[G \bar{\Pi}]=\operatorname{dim}(\tilde{d}) \\
\Leftrightarrow \operatorname{rank}\left[G\left(I-H^{+} H\right)\right]=\operatorname{dim}(d)-\operatorname{dim}(\bar{d}) \\
\Leftrightarrow \operatorname{rank}\left[G\left(I-H^{+} H\right)\right]=m-\operatorname{rank}[H],
\end{gathered}
$$

which is equivalent to (35). Furthermore, we have the following relationships:

$$
\begin{gathered}
G \bar{\Pi}\left(I-S^{+} S\right)=0 \Leftrightarrow G \bar{\Pi}\left(I-S^{+} S\right) \widetilde{\Pi}=0 \\
\Leftrightarrow\left(I-G \Pi(S \widetilde{\Pi})^{+}\left(I-H H^{+}\right) C\right) G\left(I-H^{+} H\right)=0,
\end{gathered}
$$

which is equivalent to (36) [12]. Thus, the theorem is proved.

\section{B. Observer Design}

Theorem 2: Provided that the conditions in Theorem 1 hold, the SISO design of full order is given by

$$
\begin{aligned}
\dot{z}=(\breve{A} & -L \breve{C}) z+\breve{B} \breve{u}+\breve{E}_{1} \breve{y}_{i} \\
& +\left((\breve{A}-L \breve{C}) \breve{\mathrm{E}}_{2}-\dot{\mathrm{B}}_{2}\right) \breve{y}+\mathrm{Ly}
\end{aligned}
$$

$$
\widehat{\mathrm{X}}=\mathrm{z}+\breve{\mathrm{E}}_{2} \breve{\mathrm{y}}
$$

Proof: Subject to the conditions in Theorem 1 and noting the detectability of the pair $\{\breve{A}, \breve{C}\}$, one can easily design a stable full-order state estimator for the system (29), (30) as follows:

$$
\dot{\hat{X}}=\breve{A} \widehat{X}+\breve{B} \breve{u}+\breve{E}_{1} \breve{y}_{i}+\breve{E}_{2} \dot{\grave{y}}+L(y-\breve{C} \widehat{X})
$$

where the gain matrix $L$ is chosen such that $(\breve{A}-L \breve{C})$ has eigenvalues with negative real parts. Note that, to eliminate the need to implement $\dot{\dot{y}}$ in (39), we apply the approach in [12] to transform (39) into its corresponding observer form shown in (37) and (38). This completes the proof of the theorem.

Finally, based on Theorem 2 and using (4), the estimates of the state and the unknown inputs are given as follows:

$$
\widehat{\mathrm{x}}=\left[\begin{array}{lll}
1 & 0 & 0
\end{array}\right] \widehat{\mathrm{X}}, \quad \widehat{\mathrm{d}}=\left[\begin{array}{lll}
0 & \widetilde{\mathrm{H}}^{+} & \bar{\Pi}
\end{array}\right] \widehat{\mathrm{X}}
$$

Remark 2: The observer gain matrix $L$ can be obtained by applying Kalman filtering as follows:

$$
\begin{gathered}
L=P \breve{C}^{T} R^{-1}, \\
\dot{P}=P \breve{A}^{T}+\breve{A} P-P \breve{C}^{T} R^{-1} \breve{C} P+Q,
\end{gathered}
$$

where $Q$ and $R$ are filter design parameters.

Remark 3: If matrix $H$ is of full-column rank, the SISO given by (37), (38) is reduced to the following compact form due to (13) and (14):

$$
\begin{aligned}
& \dot{z}=(\bar{A}-L \breve{C}) z+\bar{B} u+((\bar{A}-L \breve{C}) E-\dot{E}+L) y, \\
& \hat{X}=z+E y,
\end{aligned}
$$

where

$$
\begin{array}{cc}
\bar{A}=\left[\begin{array}{cc}
A & G \\
-H^{+}(\dot{C}+C A) & -H^{+}(\dot{H}+C G)
\end{array}\right], \\
\bar{B}=\left[\begin{array}{c}
B \\
-H^{+} C B
\end{array}\right], & E=\left[\begin{array}{c}
0 \\
H^{+}
\end{array}\right] .
\end{array}
$$

\section{AN ILlUSTRATIVE EXAMPLE}

In order to illustrate the usefulness of the proposed results, we considered the dynamics of a vehicle represented by the bicycle model with a roll mode as given in [15], where the system parameters of (1)-(2) can be expressed as follows:

$$
x=\left[\begin{array}{c}
\beta \\
\gamma \\
\emptyset_{v} \\
\dot{\emptyset}_{v}
\end{array}\right], \quad B=\left[\begin{array}{c}
\frac{I_{e q} c_{\alpha f}}{I_{x} m V} \\
\frac{a c_{\alpha f}}{I_{z}} \\
0 \\
\frac{h c_{\alpha f}}{I_{x}}
\end{array}\right], \quad G=\left[\begin{array}{ccc}
\frac{-g}{V} & 0 & 0 \\
0 & 0 & 0 \\
0 & 0 & 0 \\
0 & 0 & -1
\end{array}\right],
$$




$$
\begin{gathered}
A=\left[\begin{array}{cccc}
-\frac{I_{e q} C_{0}}{I_{x} m V} & -1-\frac{I_{e q} C_{1}}{I_{x} m V^{2}} & \frac{h\left(m g h-k_{r}\right)}{I_{x} V} & -\frac{h b}{I_{x} V} \\
-\frac{C_{1}}{I_{z}} & -\frac{C_{2}}{I_{z} V} & 0 & 0 \\
0 & 0 & 0 & 1 \\
-\frac{C_{0} h}{I_{x}} & -\frac{C_{1} h}{I_{x} V} & \frac{\left(m g h-k_{r}\right)}{I_{x}} & -\frac{b_{r}}{I_{x}}
\end{array}\right], \\
u=\delta, \quad C=I_{4}, \quad H=\left[\begin{array}{ccc}
0 & 0 & 0 \\
0 & 0 & 0 \\
1 & 0 & 0 \\
0 & 1 & 0
\end{array}\right], \quad d=\left[\begin{array}{c}
\emptyset_{r} \\
p_{f} \\
\dot{p}_{f}
\end{array}\right], \\
C_{0}=C_{\alpha f}+C_{\alpha r}, \quad C_{1}=a C_{\alpha f}-b C_{\alpha r}, \\
C_{2}=a^{2} C_{\alpha f}+b^{2} C_{\alpha r}, \quad I_{e q}=I_{x}+m h^{2} .
\end{gathered}
$$

In the simulation, the disturbance observer (DO) [16] and the proposed SISO in this paper were considered. Note that, the DO which is intended to augment the state vector is given as:

$$
\left[\begin{array}{c}
\dot{\mathrm{d}} \\
\dot{\varepsilon}_{\mathrm{r}}
\end{array}\right]=\left[\begin{array}{cc}
\mathrm{A}_{1}^{\mathrm{d}} & \mathrm{A}_{2}^{\mathrm{d}} \\
0 & 0
\end{array}\right]\left[\begin{array}{c}
\mathrm{d} \\
\varepsilon_{\mathrm{r}}
\end{array}\right], \quad\left[\begin{array}{ll}
\mathrm{A}_{1}^{\mathrm{d}} & \mathrm{A}_{2}^{\mathrm{d}}
\end{array}\right]=\left[\begin{array}{cccc}
0 & 1 & 0 & 1 \\
0 & 0 & 1 & 0 \\
0 & 0 & 0 & 0
\end{array}\right]
$$

where $\varepsilon_{r} \approx \dot{\emptyset}_{r}-p_{f}$. Thus, the vehicle system model used in the DO method is given as follows:

$$
\begin{gathered}
{\left[\begin{array}{c}
\dot{x} \\
\dot{d} \\
\dot{\varepsilon}_{r}
\end{array}\right]=\left[\begin{array}{ccc}
A & G & 0 \\
0 & A_{1}^{d} & A_{2}^{d} \\
0 & 0 & 0
\end{array}\right]\left[\begin{array}{l}
x \\
d \\
\varepsilon_{r}
\end{array}\right]+\left[\begin{array}{l}
B \\
0 \\
0
\end{array}\right] u} \\
y=\left[\begin{array}{lll}
C & H & 0
\end{array}\right]\left[\begin{array}{lll}
x^{T} & d^{T} & \varepsilon_{r}
\end{array}\right]^{T}
\end{gathered}
$$

On the other hand, due to that $S_{i}=0$ for all $i \geq 1$, we obtain that the time derivative of the roll rate $\left(\dot{p}_{f}\right)$ is unestimable through the CSISE method. To remedy this unestimation problem, we further assume that the dynamics of $\dot{p}_{f}$ due to the road changes is the result of white noise forcing $\ddot{p}_{f}[16]$, which yields $\ddot{p}_{f}=0$. Thus, the vehicle system model used in the SISO is given as follows:

$$
\begin{gathered}
{\left[\begin{array}{c}
\dot{x} \\
\ddot{p}_{f}
\end{array}\right]=\left[\begin{array}{cc}
A & G_{2} \\
0 & 0
\end{array}\right]\left[\begin{array}{c}
x \\
\dot{p}_{f}
\end{array}\right]+\left[\begin{array}{c}
B \\
0
\end{array}\right] u+\left[\begin{array}{c}
G_{1} \\
0
\end{array}\right] \breve{d}} \\
y=\left[\begin{array}{ll}
C & 0
\end{array}\right]\left[\begin{array}{ll}
x^{T} & \dot{p}_{f}^{T}
\end{array}\right]^{T}+H_{1} \breve{d}
\end{gathered}
$$

where

$$
G=\left[\begin{array}{ll}
G_{1} & G_{2}
\end{array}\right], \quad H_{1}=H T^{T}, \quad \breve{d}=T d, \quad T=\left[\begin{array}{lll}
1 & 0 & 0 \\
0 & 1 & 0
\end{array}\right] .
$$

Notice that the above matrix $H_{1}$ is of full-column rank, and thus, the compact observer form in Remark 3 is applied to achieve the CSISE.
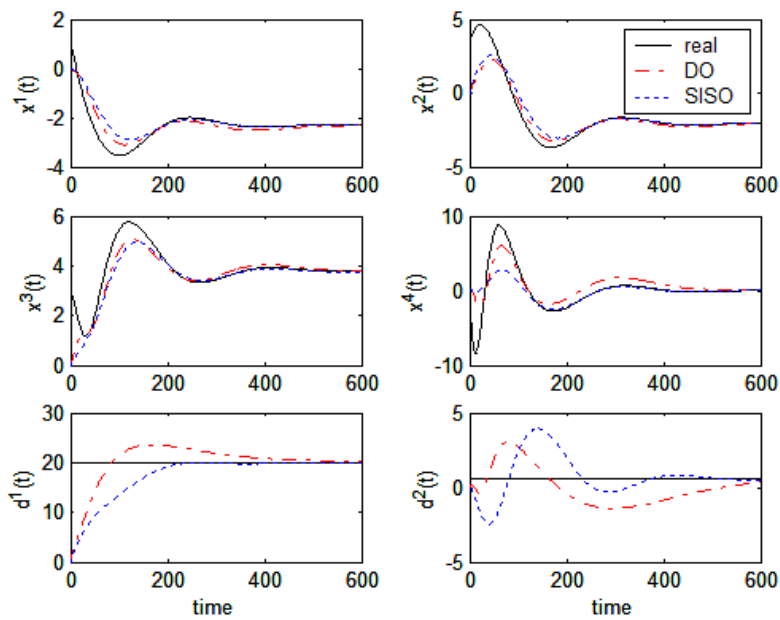

FIGURE I. STATE AND UNKNOWN INPUTS ESTIMATES OF THE DO AND THE SISO

This study illustrates the state and unknown inputs estimates of the DO and the SISO in Figure 1, where the fourth order Runge-Kutta simulation method is used to obtain the estimates. From the figure, we may obtain the following conclusions.

(1) Both the DO and the SISO can successfully estimate the state and the unknown inputs; the estimator complexity of the latter is usually smaller than that of the former.

(2) The unknown input filtering performance of the SISO is slightly outperform that of the DO. This is due to the fact that, in the DO the implicit unknown inputs model (41) is assumed, which may not reflect the true situation. On the other hand, in the proposed SISO no unknown inputs model is assumed; instead, a fictitious unknown inputs dynamics (see Remark 1 for details), which is obtained by measurements and possible measurement derivatives, is used.

(3) Provided that the existence conditions in Theorem 1 hold, the proposed SISO serves as a useful and compact estimator structure to achieve the CSISE.

\section{CONCLUSIONS}

The design of simultaneous input and state observers (SISOs) for linear time-varying continuous-time systems with unknown inputs is addressed. Using a novel equivalent augmented state system description, the addressed CSISE problem can easily be dealt with using existing literature results. The existence conditions of the proposed SISO is given. Furthermore, an application of the proposed results to achieve a practical vehicle state and unknown disturbances estimation is also presented to illustrate the contribution of the paper.

\section{ACKNOWLEDGMENT}

This work was supported by the Ministry of Science and Technology, R.O.C. under Grant MOST 104-2221-E-233-004.

\section{REFERENCES}

[1] M. Hou and R. J. Patton, "Input observability and input reconstruction," Automatica, 34, pp. 789-794, 1998. 
[2] R. A. Chavan, K. Fitch, and H. J. Palanthandalam-Madapusi, "Recursive input reconstruction with a delay," in Proceedings of the American Control Conference, pp. 628-633, 2014.

[3] G. De Nicolao, G. Sparacino, and C. Cobelli, "Nonparametric input estimation in physiological systems: Problems, methods, and case studies," Automatica, 33, pp. 851-870, 1997.

[4] M. S. Phatak and N. Viswanadham, "Autuator fault detection and isolation in linear systems," International Journal of Systems Science, 19 pp. 2593-2603, 1989 .

[5] Z. Gao and D. W. C. Ho, "State/noise estimator for descriptor systems with applications to sensor fault diagnosis," IEEE Transactions on Signal Processing, 54, pp. 1316-1326, 2006.

[6] S. Gillijns and B. De Moor, "Unbiased minimum-variance input and state estimation for linear discrete-time systems with direct feedthrough," Automatica, 43, pp. 934-937, 2007.

[7] C.-S. Hsieh, "Extension of unbiased minimum-variance input and state estimation for systems with unknown inputs," Automatica, 45, pp. 21492153, 2009.

[8] C.-S. Hsieh, "Unbiased minimum-variance input and state estimation for systems with unknown inputs: A system reformation approach," Submitted to Automatica, 2016.

[9] C.-S. Hsieh, "State estimation for descriptor systems via the unknown input filtering method," Automatica, 49, pp. 1281-1286, 2013.

[10] M. Hou and P. C. Müller, "Design of observers for linear systems with unknown inputs," IEEE Transactions on Automatic Control, 37, pp. 871875, 1992.

[11] F. Yang and R. W. Wilde, "Observers for linear systems with unknown inputs," IEEE Transactions on Automatic Control, 33, pp. 677-681, 1988

[12] M. Hou and P. C. Müller, "Disturbance decoupled observer design: A unified viewpoint," IEEE Transactions on Automatic Control, 39, pp. 1338-1341, 1994.

[13] S.-H. Wang, E. J. Davison, and P. Dorato, "Observing the states of systems with unmeasurable disturbances," IEEE Transactions on Automatic Control, pp. 716-717, 1975.

[14] S. Z. Yong, M. Zhu, and E. Frazzoli, "Simultaneous input and state estimation for Linear time-invariant continuous-time stochastic systems," in Proceedings of the American Control Conference, pp. 2511-2518, 2015.

[15] C.-S. Hsieh, S. S.-D. Xu, and W.-T. Lin, "Unknown input filtering for vehicle state and road bank angle estimation," in Proceedings of the 1th International Conference on Engineering and Science, Yilan, Taiwan, 810 July, 2016

[16] J. Ryu and J. C. Gerdes, "Estimation of vehicle roll and road bank angle," in Proceedings of the American Control Conference, pp. 2110-2115, 2004 